\title{
EXPLICIT EXPONENTIAL FINITE DIFFERENCE SCHEME FOR 1D NAVIER-STOKES EQUATION WITH TIME DEPENDENT PRESSURE GRADIENT
}

\author{
M. A. K. Azad ${ }^{1, *}$ and L. S. Andallah ${ }^{2}$ \\ ${ }^{1}$ Department of Mathematics, Shaheed Begum Sheikh Fazilatun Nessa Mujib Govt. College,Dhaka \\ ${ }^{2}$ Department of Mathematics, Jahangirnagar University, Savar, Dhaka, Bangladesh \\ *Corresponding author: akazad2034@gmail.com
}

Received 25.04.2016 Accepted 01.10.2016

\begin{abstract}
In this paper, numerical technique for solving the one-dimensional (1D) unsteady, incompressible Navier-Stokes equation (NSE) is presented. The governing time dependent non-linear partial equation is reduced to non-linear partial differential equation named as viscous Burgers' equation by introducing Orlowski and Sobczyk transformation (OST). An explicit exponential finite difference scheme (Expo FDS) has been used for solving reduced 1D NSE. The accuracy of the method has been illustrated by taking two numerical examples. Results are compared with the analytical solutions and those obtained based on the numerical results of reduced 1D NSE as Burgers' equation. The accuracy and numerical feature of convergence of the Expo FDS is presented by estimating their error norms. Excellent numerical results indicate that the proposed numerical technique is efficient admissible with efficient accuracy for the numerical solutions of the NSE.
\end{abstract}

Key-words: Navier-Stokes equation, Burgers' equation, Explicit exponential finite difference scheme

\section{Introduction}

The NSEs are one of the most important, beautiful, potentially lucrative governing equations in fluid dynamics which describe the motion of fluid substances. These equations arise from applying Newton's second law to fluid motion. Solutions and smoothness of full NSEs remain one of the open millennium dollar challenge in Mathematical Physics. Renowned Physicist and Mathematician like Stephen Montogomery-Smith,a mathematician at the University of Missouri in Columbia, who has been tackling the equation since 1995 and Otelbaev- a member of the Kazakh Academy of Sciences who has been working on the problem for 30 years. In order to understand the non-linear phenomenon of NSE, one needs to study 1D NSE as a simplification of full NSEs. Since 1D NSE includes pressure-gradient term, advection and diffusion terms, it incorporates all main mathematical features of the full NSEs. It is thus an important governing equation in the research arena of computational fluid dynamics. Applying OST we have reduced 1D NSE to viscous Burgers' equation and we have to solve viscous Burgers' equation numerically. So a 
number of analytical and numerical studies on 1D NSE as well as 1D viscous Burgers' equation have been conducted to solve the governing equation analytically and numerically. Rusli et al. [1] solved 2D NSEs numerically using a finite difference based method which essentially took advantage of the best features of two well-established numerical formulations. Azad and Andallah [2] presented analytical solution of one dimensional Navier-Stokes Equations for a time dependent exponentially decreasing pressure gradient term using Orlowski and Sobczyk transformation and Cole-Hopf transformation. Azad andAndallah [3] studied on numerical solutions of onedimensional Navier-Stokes equation using explicit finite difference scheme. Young and McDonough [4] presented exact solutions of onedimensional Burgers' equation which is analogous to one-dimensional Navier-Stokes equation. Orlowski and Sobczyk [5] discovered a transformation by which one can transform 1D NSE to 1D Burgers' equation. The explicit exponential finite difference method has originally developed by Bhattacharya [6] for the solution of heat equation. Bhattacharya [7] used explicit exponential finite difference method for the solution of Burgers' equation. Bahadir [8] solved the $\mathrm{KdV}$ equation by using the exponential finite difference technique. Implicit exponential finite difference method and fully implicit exponential finite difference method was applied to Burgers' equation by Inan and Bahadir [9]. Kutlay and Bahadir [10] also proposed finite difference solution of the finite difference approximations based on the standard explicit method to the one-dimensional Burgers' equation.

In this paper, we study Expo FDS to get the numerical solutions of reduced 1D NSE. We solve the governing equation numerically with the help of solutions of reduced 1D NSE and applying back substitution from OST. We find error norms to determine the accuracy of the numerical scheme. Finally, we will compare our numerical solutions with other available results to verify the effectiveness of our numerical techniques.

\section{Governing Equation}

Using the dimensionless variable quantities as mentioned in Rusli et al. [1], Azad and Andallah [2],

$$
x=\frac{x^{*}}{L}, u=\frac{u^{*}}{V}, p=\frac{p^{*}}{\rho V^{2}}, t=\frac{t^{*} V}{L}
$$

We consider the1D NSE in non-dimension form Azad \& Andallah [2], [3], Yang and McDonough [4] as

$$
u_{t}+u u_{x}+p_{x}=\frac{1}{R e} u_{x x}
$$

Where $x \in(a, b), t \in\left(t_{0}, t_{f}\right)$.

With $u(x, t)$ denotes velocity, subscripts denote partial differentiation. We consider pressure gradient as a function of $\mathrm{t}$ of the form ${ }^{-p_{X}}=f(t)$.

As a result equation $(1)$ can be reads as 
$u_{t}+u u_{x}-\frac{1}{R_{e}} u_{x x}=f(t)$

Where $\frac{1}{\operatorname{Re}}=\frac{y}{V L}$,

In order to determine analytical solution of equation (2) first we reduce the equation to Burgers' equation by using OST discovered by Orlowski and Sobczyk [5]. Then solving Burgers' equation analytically we obtain analytical solution of 1D NSE (2) by using inverse OST.

The OST is defined as

$x^{\prime}=x-e^{2}(t) t^{\prime}=t, u^{\prime}\left(x^{\prime}, t^{\prime}\right)=u(x, t)-W(t)$

$W(t)=\int f(r) d \tau=[F(t)]_{0}^{t}=F(t)-F(0)$

$\varphi(t)=\int_{0}^{s} W(t) d \tau=\int_{0}^{c}[F(t)-F(0)] d t=G(t)-t F(0)-G(0)$

$t^{\prime}=t$

$u^{\prime}\left(x^{\prime}, t^{\prime}\right)=u(x, t)-F(t)+F(0)$

Now

$$
\begin{aligned}
& \frac{\partial u}{\partial t}=\frac{\partial u^{\prime}}{\partial x^{\prime}} \text { 固 EMBED Equation. } 3 \text { E } \\
& \frac{\partial u}{\partial x}=\frac{\partial u^{\prime}}{\partial x^{\prime}} \\
& \frac{\partial^{2} u}{\partial x^{2}}=\frac{\partial^{2} u^{\prime}}{\partial x^{\prime 2}} \\
& \frac{\partial u^{\prime}}{\partial t^{\prime}}+\frac{u^{\prime} \partial u^{\prime}}{\partial x^{\prime}}=\frac{1}{R e} \frac{\partial^{2} u^{\prime}}{\partial x^{\prime 2}}
\end{aligned}
$$

\section{Problem 1}

We solve reduced 1D NSE (8) and the initial condition

$u^{\prime}\left(x^{\prime}, 0\right)=\sin \left(\pi x^{\prime}\right), 0<x^{\prime}<1$

With the boundary conditions

$$
u^{\prime}\left(0, t^{\prime}\right)=u^{\prime}\left(1, t^{\prime}\right)=0, t^{\prime}>0
$$

And the exact solution given by

$u^{\prime}\left(x^{\prime}, t^{\prime}\right)=\frac{2 \pi \sum_{n=1}^{\infty} A_{n} \exp \left(-\frac{n^{2} \pi^{2} t^{\prime}}{R e}\right) n \sin \left(n \pi x^{\prime}\right)}{\operatorname{Re}\left\{A_{0}+\sum_{n=1}^{\infty} A_{n} \exp \left(-\frac{n^{2} \pi^{2} t^{\prime}}{R e}\right) \cos \left(n \pi x^{\prime}\right)\right\}}$

With 


$$
\begin{aligned}
& A_{0}=\int_{0}^{1} \exp \left\{-\left(\frac{2 \pi}{R e}\right)^{-1}\left[1-\cos \left(\pi x^{\prime}\right)\right]\right\} d x^{\prime} \\
& A_{n}=\mathbf{Z} \int_{0}^{1} \exp \left\{-\left(\frac{2 \pi}{R_{e}}\right)^{-2}\left[1-\cos \left(\pi x^{\prime}\right)\right]\right\} \cos \left(n \pi x^{\prime}\right) d x^{\prime}, n=1,2,3, \ldots
\end{aligned}
$$

\section{Problem 2}

The initial condition for the current problem is

$u^{\prime}\left(x^{\prime}, 0\right)=4 x^{\prime}\left(1-x^{\prime}\right), 0<x^{\prime}<1$

And the boundary conditions

$u^{\prime}\left(0, t^{\prime}\right)=u^{\prime}\left(1, t^{\prime}\right)=0, t^{\prime}>0$

And the exact solution given by

$u^{\prime}\left(x^{\prime}, t^{\prime}\right)=\frac{2 \pi \sum_{n=1}^{\infty} A_{n} \exp \left(-\frac{n^{2} \pi^{2} t^{\prime}}{R e}\right) n \sin \left(n \pi x^{\prime}\right)}{\operatorname{Re}\left\{A_{0}+\sum_{n=1}^{\infty} A_{n} \exp \left(-\frac{n^{2} \pi^{2} t^{\prime}}{R e}\right) \cos \left(n \pi x^{\prime}\right)\right\}}$

With

$$
\begin{aligned}
& A_{0}=\int_{0}^{1} \operatorname{\theta xp}\left\{-x^{\prime 2}\left(\frac{3}{R e}\right)^{-1}\left(3-2 x^{\prime}\right)\right\} d x^{\prime \prime} \\
& A_{n}=2 \int_{0}^{1} \operatorname{Exp}\left\{-x^{\prime 2}\left(\frac{3}{R e}\right)^{-1}\left(3-2 x^{\prime}\right)\right\} \cos \left(n \pi x^{\prime}\right) d x^{\prime}, n=1,2,3, \ldots
\end{aligned}
$$

\section{Explicit Exponential Finite Difference Scheme (Expo FDS)}

We assume that $H\left(u^{\prime}\right)$ denote to any continuous differential function. Multiplying equation (2) by the derivative of $\mathrm{H}$ lead to the following equation:

$\frac{\frac{\partial H}{\partial u^{\prime}} \partial u^{\prime}}{\partial t^{\prime}}=\frac{\partial H}{\partial u^{\prime}\left[-u^{\prime} u_{x}^{\prime \prime}+\frac{1}{R e} \frac{\partial^{2} u^{\prime}}{\partial x^{\prime 2}}\right]}$

And

$\frac{\partial H}{\partial t^{\prime}}=\frac{1}{u^{\prime}\left[-u^{\prime} u_{x}^{\prime \prime}+\frac{1}{R e} \frac{\partial^{2} u^{\prime}}{\partial x^{\prime 2}}\right]}$

Where ${ }^{H}=$ ints',

Using the usual forward difference replacement to $\frac{\partial H}{\partial t^{\prime}}$ we obtain the finite-difference representation of equation (2) as

$\frac{H_{i}^{n+1}-H_{i}^{n}}{k}=\frac{1}{u^{\prime}}\left[-u_{i}^{\prime n} \frac{u_{i+1}^{\prime n}-u_{i-1}^{\prime n}}{2 h}+\frac{1}{R e}\left\{\frac{u_{i+1}^{m}-2 u_{i}^{m}+u_{i-2}^{\prime n}}{h^{2}}\right\}\right]$ 
Where $h \cong \Delta x^{\prime}, k \cong \Delta t^{\prime}$ are spatial and time step sizes respectively.

As a result Expo FDS takes the form

$u_{i}^{\prime n+1}=u_{i}^{\prime n} \exp \left[\frac{k}{u_{i}^{\prime \pi}}\left\{-u_{i}^{\prime n} \frac{u_{i+2}^{\prime \pi}-u_{i-1}^{\prime n}}{2 h}+\frac{1}{R e}\left(\frac{u_{i+1}^{\prime \pi}-2 u_{i}^{\prime n}+u_{i-2}^{\prime \pi}}{h^{2}}\right)\right\}\right]$

By using OST we obtain numerical solution by using Expo FDS and OST as

$u_{i}^{n+1}=u_{i}^{\prime n} \exp \left[\frac{k}{u_{i}^{\prime n}}\left\{-u_{i}^{\prime n} \frac{u_{i+1}^{\prime n}-u_{i-1}^{\prime n}}{2 h}+\frac{1}{R e}\left(\frac{u_{i+1}^{\prime n}-2 u_{i}^{\prime n}+u_{i-1}^{\prime n}}{h^{2}}\right)\right\}\right]+F\left(t_{n}\right)-F(0)$

If we take $-p_{z}=f(t)=e^{-a t-b}$ then Expo FDS yields

$$
u_{i}^{n+1}=\frac{1}{a} e^{-b}-\frac{1}{a} e^{-a r_{n}}+u_{i}^{\prime n} \exp \left[\frac { k } { u _ { i } ^ { \prime n } } \left\{-u_{i}^{\prime n} \frac{u_{i+1}^{\prime n}-u_{i-1}^{\prime \pi}}{2 h}+\frac{1}{R e}\left(\frac{u_{i+1}^{\prime \pi}-2 u_{i}^{\prime \pi}+u_{i}^{\prime n}}{h^{2}}\right.\right.\right.
$$

\section{Numerical Results and Discussions}

In order to show how good the numerical solutions for problem 1exhibit the correct physical characteristic we only give the graphs in figures 1-5 which show the numerical solutions at different times for $\operatorname{Re}=10$ taking $h=0.0125, k=0.001, a=2, b=4$.

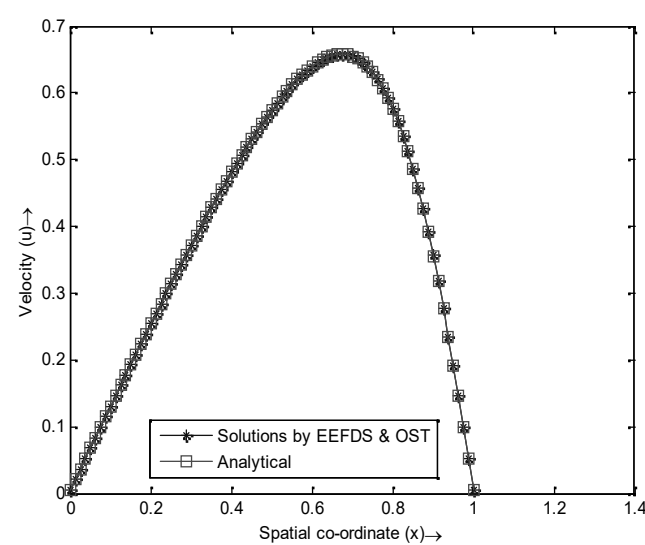

Figure 1: Comparisons of numerical and analytical solutions at $t=0.4$.

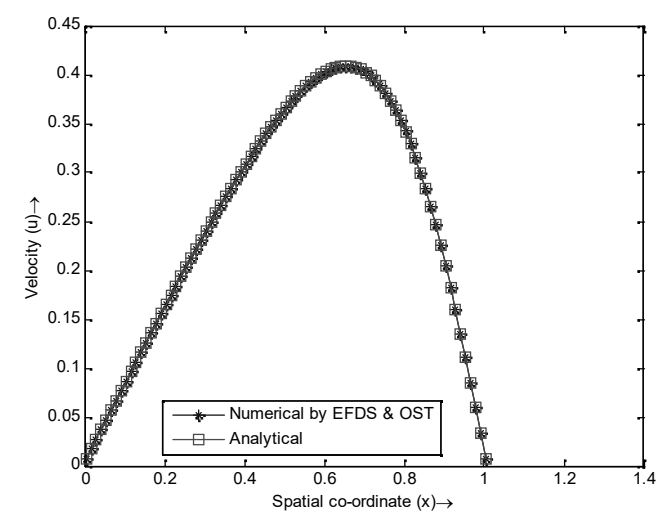

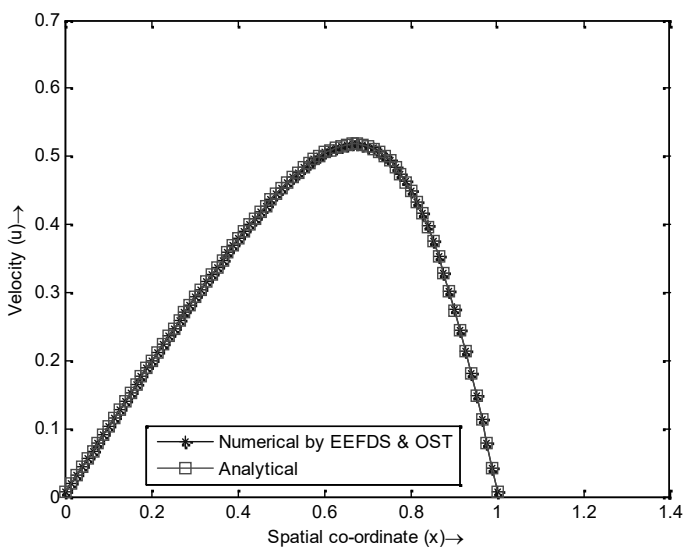

Figure 2:Comparisons of numerical and analytical solutions at $t=0.6$.

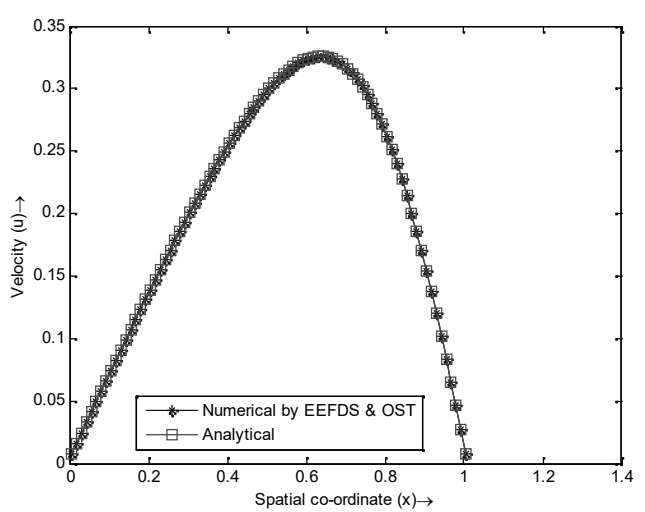


Figure 3: Comparisons of numerical and analytical solutions at $t=0.8$.
Figure 4: Comparisons of numerical and analytical solutions at $t=1.0$.

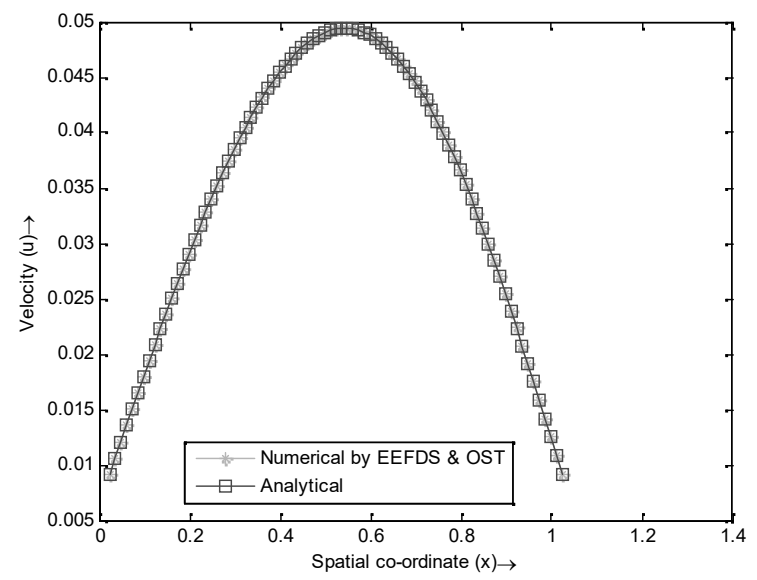

Figure 5:Comparisons of numerical and analytical solutions at $t=3,0$.

For problem 1, numerical solutions by Expo FD Sand analytical solutions obtained to 1D NSE for $\operatorname{Re}=10$ are displaying at different times in figures 1-5. It is clearly seen that the numerical solutions obtained by Expo FDS and analytical solutions are well-suited. From graphical representation we observe that it is almost impossible to distinguish due to the closeness of our numerical solutions to analytical solutions.

Table 1: Comparison of the numerical values of $u^{\prime}$ taking $h=0.0125, k=0.001, \operatorname{Re}=10, t^{\prime}=3.0$ and $x^{\prime}=1.0$.

\begin{tabular}{|c|c|c|c|c|c|c|c|c|c|}
\hline$x$ & $t^{2}$ & $\begin{array}{c}\text { Numerical } \\
\text { values of } \\
u \\
\text { By Expo } \\
\text { FDS }\end{array}$ & $\begin{array}{c}\text { Numerical } \\
\text { values of } \\
\text { By Kutlay } \\
\text { et al. [10] }\end{array}$ & $\begin{array}{c}\text { Exact } \\
\text { values of } \\
u^{-}\end{array}$ & $x$ & t & $\begin{array}{c}\text { Numerical } \\
\text { values of } \\
u \\
\text { By Expo } \\
\text { FDS }\end{array}$ & $\begin{array}{c}\text { Numerical } \\
\text { values of } u \\
\text { Based on Kutlay } \\
\text { et al. [10] }\end{array}$ & $\begin{array}{c}\text { Analytical } \\
\text { values of } u\end{array}$ \\
\hline \multirow[t]{5}{*}{0.25} & 0.4 & 0.30885 & 0.30891 & 0.30889 & 0.27 & 0.4 & 0.31388 & 0.31395 & 0.31393 \\
\hline & 0.6 & 0.24072 & 0.24075 & 0.24074 & & 0.6 & 0.24712 & 0.24715 & 0.24713 \\
\hline & 0.8 & 0.19570 & 0.19568 & 0.19568 & & 0.8 & 0.20301 & 0.20299 & 0.20297 \\
\hline & 1.0 & 0.16262 & 0.16257 & 0.16256 & & 1.0 & 0.17054 & 0.17049 & 0.17048 \\
\hline & 3.0 & 0.02727 & 0.02720 & 0.02720 & & 3.0 & 0.03640 & 0.03634 & 0.03634 \\
\hline \multirow[t]{5}{*}{0.50} & 0.4 & 0.56984 & 0.56964 & 0.56963 & 0.52 & 0.4 & 0.57488 & 0.57469 & 0.57467 \\
\hline & 0.6 & 0.44744 & 0.44721 & 0.44721 & & 0.6 & 0.45384 & 0.45361 & 0.45385 \\
\hline & 0.8 & 0.35951 & 0.35924 & 0.35924 & & 0.8 & 0.36682 & 0.36655 & 0.36652 \\
\hline & 1.0 & 0.29221 & 0.29192 & 0.29192 & & 1.0 & 0.30013 & 0.29984 & 0.29985 \\
\hline & 3.0 & 0.04030 & 0.04021 & 0.04021 & & 3.0 & 0.04944 & 0.04935 & 0.04934 \\
\hline
\end{tabular}




\begin{tabular}{|cccccccccc|}
\hline 0.75 & 0.4 & 0.62667 & 0.62542 & 0.62544 & 0.77 & 0.4 & 0.63170 & 0.63046 & 0.63041 \\
& 0.6 & 0.48835 & 0.48721 & 0.48721 & & 0.6 & 0.49475 & 0.49361 & 0.49360 \\
& 0.8 & 0.37486 & 0.37392 & 0.37392 & & 0.8 & 0.38216 & 0.38123 & 0.38123 \\
& 1.0 & 0.28820 & 0.28748 & 0.28747 & & 1.0 & 0.29612 & 0.29540 & 0.29539 \\
& 3.0 & 0.02985 & 0.02977 & 0.02977 & & 3.0 & 0.03898 & 0.03891 & 0.03890 \\
\hline
\end{tabular}

Table 1 depicts comparisons among the numerical values of $u^{\prime \prime}$ for our present study and numerical values obtained by by Kutlay et al. [10] and analytical solutions taking different nodes, At $x^{\prime}=0.25, t^{\prime}=0.4,0.6,0.8,1.0,3.0 \& x^{\prime}=0.50, t^{\prime}=0.4,0.6,0.8,3.0$ we see up to three places agreement. In other nodes we also observe a nice agreement among our numerical solutions with numerical solutions obtained based on Kutlay et al. [10] and analytical solutions for $\boldsymbol{u}^{\prime}$.

Table 2 : Comparison of the numerical values of $u^{\prime}$ with available published works at different times taking $R e=10, h=0.0125,=10^{-4}, a=2$ and $b=4$.

\begin{tabular}{|c|c|c|c|c|c|c|c|}
\hline $\mathrm{x}^{\prime}$ & $\mathrm{t}^{\prime}$ & $\begin{array}{c}\text { RHC By } \\
\text { Gülsu\&Öziş } \\
{[11]}\end{array}$ & $\begin{array}{c}\text { RPA By } \\
\text { Gülsu[12] }\end{array}$ & $\begin{array}{c}\text { I-EFDM } \\
\text { By Inan } \\
\text { Bilge [15] }\end{array}$ & $\begin{array}{c}\text { FI-EFDM } \\
\text { By Inan } \\
\text { Bilge [13] }\end{array}$ & $\begin{array}{c}\text { Present } \\
\text { Work By } \\
\text { Expo FDS }\end{array}$ & Analytical \\
\hline 0.25 & 0.4 & 0.317062 & 0.308776 & 0.308936 & 0.308962 & 0.308902 & 0.308894 \\
& 0.6 & 0.248472 & 0.240654 & 0.240775 & 0.240795 & 0.240750 & 0.240739 \\
& 0.8 & 0.202953 & 0.195579 & 0.195709 & 0.195725 & 0.195691 & 0.195676 \\
& 1.0 & 0.169527 & 0.162513 & 0.162599 & 0.162612 & 0.162585 & 0.162565 \\
0.5 & 0.4 & 0.583408 & 0.569527 & 0.569727 & 0.569762 & 0.569695 & 0.569632 \\
& 0.6 & 0.461714 & 0.447117 & 0.447307 & 0.447337 & 0.447275 & 0.447206 \\
& 0.8 & 0.373800 & 0.359161 & 0.359343 & 0.359368 & 0.359313 & 0.359236 \\
& 1.0 & 0.306184 & 0.291843 & 0.292026 & 0.292046 & 0.291996 & 0.291916 \\
0.75 & 0.4 & 0.638847 & 0.625341 & 0.625659 & 0.625676 & 0.625695 & 0.625438 \\
& 0.6 & 0.506429 & 0.487089 & 0.487495 & 0.487513 & 0.487480 & 0.487215 \\
& 0.8 & 0.393565 & 0.373827 & 0.374187 & 0.374203 & 0.374150 & 0.373922 \\
& 1.0 & 0.305862 & 0.029726 & 0.287700 & 0.287714 & 0.287658 & 0.287447 \\
\hline
\end{tabular}

RHC- Restrictive Hopf-Cole method, RPA- Restrictive Pade Approximation

Table 3 : Comparison of the numerical values of $u$ with available published works at different times taking $R e=10, h=0.0125, k=10^{-4}, \underline{a}=2, \underline{b}=4$.

\begin{tabular}{|c|c|c|c|c|c|c|c|}
\hline$x$ & $\tau$ & $\begin{array}{c}\text { Based on RHC } \\
\text { By Gülsu\&Öziş } \\
[11])\end{array}$ & $\begin{array}{c}\text { Based on } \\
\text { RPA By } \\
\text { Gülsu[12] }\end{array}$ & $\begin{array}{c}\text { Based on I- } \\
\text { EFDM By } \\
\text { Inan Bilge } \\
{[13]}\end{array}$ & $\begin{array}{c}\text { Based on } \\
\text { FI-EFDM } \\
\text { By Inan } \\
\text { Bilge [13] }\end{array}$ & $\begin{array}{c}\text { Present } \\
\text { Work By } \\
\text { Expo FDS }\end{array}$ & Analytical \\
\hline 0.2511 & 0.4 & 0.322104 & 0.313819 & 0.313979 & 0.314005 & 0.313944 & 0.313937 \\
0.2523 & 0.6 & 0.254872 & 0.247054 & 0.247175 & 0.247195 & 0.247149 & 0.247139 \\
0.2537 & 0.8 & 0.210262 & 0.202888 & 0.203018 & 0.203034 & 0.202999 & 0.202985 \\
0.2552 & 1.0 & 0.177445 & 0.170431 & 0.170517 & 0.170530 & 0.170502 & 0.170483 \\
0.5011 & 0.4 & 0.588451 & 0.574570 & 0.574770 & 0.574805 & 0.574737 & 0.574675 \\
0.5023 & 0.6 & 0.468114 & 0.453517 & 0.453707 & 0.453737 & 0.453674 & 0.453606
\end{tabular}




\begin{tabular}{|llllllll|}
0.5037 & 0.8 & 0.381109 & 0.366470 & 0.366652 & 0.366677 & 0.366621 & 0.366545 \\
0.5052 & 1.0 & 0.314102 & 0.299761 & 0.299944 & 0.299964 & 0.299914 & 0.299834 \\
0.7511 & 0.4 & 0.643890 & 0.630384 & 0.630702 & 0.630719 & 0.630738 & 0.630481 \\
0.7523 & 0.6 & 0.512829 & 0.493489 & 0.493895 & 0.493913 & 0.493879 & 0.493615 \\
0.7537 & 0.8 & 0.400874 & 0.381136 & 0.381496 & 0.381512 & 0.381459 & 0.381231 \\
0.7552 & 1.0 & 0.313780 & 0.305178 & 0.295618 & 0.295632 & 0.287658 & 0.295392 \\
\hline
\end{tabular}

Table 2 and 3 demonstrate that the obtained solutions by our present numerical techniques (using Expo FDS and OST) achieve suitable accuracy with analytical solutions and numerical results obtained based on other methods like RHC implemented by GülsuandÖziş[11], RPA by Gülsu[12], I-EFD Mand FI-EFDM implemented by InanandBahadir[13]and OST.

Table 4 : Comparison of the numerical solutions with analytical solutions at different times for $R e=1.0, h=0.025, k=0.0001, a=2$ and $b=4$ for problem 2.

\begin{tabular}{|c|c|c|c|c|c|c|c|}
\hline$x^{\prime}$ & $t^{\prime}$ & \multicolumn{2}{|c|}{$\begin{array}{c}\text { Numerical solution of } \\
\text { reduced NSE }\left(u^{\prime}\right)\end{array}$} & $x$ & $\tau$ & \multicolumn{2}{c|}{$\begin{array}{c}\text { Numerical solution of } \\
\text { NSE }(u)\end{array}$} \\
\hline & & Expo FDS & Analytical & & & Expo FDS & Analytical \\
\hline 0.25 & 0.01 & 0.66009 & 0.66006 & 0.25000 & 0.01 & 0.6603 & 0.66024 \\
& 0.05 & 0.42637 & 0.42629 & 0.25002 & 0.05 & 0.42724 & 0.42716 \\
& 0.10 & 0.26159 & 0.26148 & 0.25009 & 0.10 & 0.26325 & 0.263140 \\
& 0.15 & 0.16159 & 0.16148 & 0.25019 & 0.15 & 0.16396 & 0.16385 \\
& 0.25 & 0.06116 & 0.06109 & 0.25049 & 0.25 & 0.06477 & 0.06469 \\
0.5 & 0.01 & 0.91976 & 0.91972 & 0.50000 & 0.01 & 0.91994 & 0.91990 \\
& 0.05 & 0.62825 & 0.62808 & 0.50002 & 0.05 & 0.62912 & 0.62895 \\
& 0.10 & 0.38362 & 0.38342 & 0.50009 & 0.10 & 0.38528 & 0.38508 \\
& 0.15 & 0.23424 & 0.23406 & 0.50019 & 0.15 & 0.23661 & 0.23643 \\
& 0.25 & 0.08735 & 0.08723 & 0.50049 & 0.25 & 0.09095 & 0.09083 \\
& 0.75 & 0.68370 & 0.68364 & 0.75000 & 0.01 & 0.68388 & 0.68382 \\
& 0.05 & 0.46542 & 0.46525 & 0.75002 & 0.05 & 0.46629 & 0.46612 \\
& 0.10 & 0.28175 & 0.28157 & 0.75009 & 0.10 & 0.28340 & 0.28323 \\
& 0.15 & 0.16988 & 0.16974 & 0.75019 & 0.15 & 0.17226 & 0.17211 \\
& 0.25 & 0.06237 & 0.06229 & 0.75049 & 0.25 & 0.06597 & 0.06589 \\
\hline
\end{tabular}

Table 5 : Comparison of the numerical solutions with analytical solutions at different times for $\boldsymbol{t}=\mathbf{3 . 0}$, Re $=100, h=0.0125, k=0.001, a=2$ and $b=4$ for problem 2.

\begin{tabular}{|c|c|c|c|c|c|c|c|}
\hline$x^{\prime}$ & $t^{\prime}$ & \multicolumn{2}{|c|}{$\begin{array}{c}\text { Numerical solution of } \\
\text { reduced NSE }\left(u^{\prime}\right)\end{array}$} & $x$ & $\tau$ & \multicolumn{2}{c|}{$\begin{array}{c}\text { Numerical solution of } \\
\text { NSE }(u)\end{array}$} \\
\hline & & Expo FDS & Analytical & & & Expo FDS & Analytical \\
\hline 0.25 & 0.4 & 0.36211 & 0.36226 & 0.25114 & 0.4 & 0.36714 & 0.36730 \\
& 0.6 & 0.28190 & 0.28204 & 0.25229 & 0.6 & 0.28830 & 0.28844 \\
& 0.8 & 0.23034 & 0.23045 & 0.25367 & 0.8 & 0.23764 & 0.23776 \\
& 1.0 & 0.19460 & 0.19469 & 0.25520 & 1.0 & 0.20251 & 0.20261 \\
& 3.0 & 0.07611 & 0.07613 & 0.27291 & 3.0 & 0.08524 & 0.08527 \\
0.5 & 0.4 & 0.68367 & 0.68368 & 0.50114 & 0.4 & 0.68870 & 0.68872 \\
& 0.6 & 0.54818 & 0.54832 & 0.50229 & 0.6 & 0.55458 & 0.55472
\end{tabular}




\begin{tabular}{|c|c|c|c|c|c|c|c|}
\hline \multirow{8}{*}{0.75} & 0.8 & 0.45356 & 0.45371 & 0.50367 & 0.8 & 0.46086 & 0.46102 \\
\hline & 1.0 & 0.38553 & 0.38568 & 0.50520 & 1.0 & 0.39344 & 0.39360 \\
\hline & 3.0 & 0.15213 & 0.15218 & 0.52291 & 3.0 & 0.16127 & 0.16132 \\
\hline & 0.4 & 0.92123 & 0.92050 & 0.75114 & 0.4 & 0.92626 & 0.92554 \\
\hline & 0.6 & 0.78317 & 0.78299 & 0.75229 & 0.6 & 0.78957 & 0.78939 \\
\hline & 0.8 & 0.66267 & 0.66272 & 0.75367 & 0.8 & 0.66998 & 0.67003 \\
\hline & 1.0 & 0.56919 & 0.56932 & 0.75520 & 1.0 & 0.57711 & 0.57724 \\
\hline & 3.0 & 0.22770 & 0.2277 & 0.77291 & 3.0 & 0.23683 & 0.23688 \\
\hline
\end{tabular}

Table 4 and 5 display numerical solutions implemented by Expo FDS to problem 2. It is clearly observed that both numerical predictions are reasonably in good agreement with analytical solutions. In order to show how the numerical solutions of problem 2 obtained with Expo FDS, we give the graph in figure 6 .

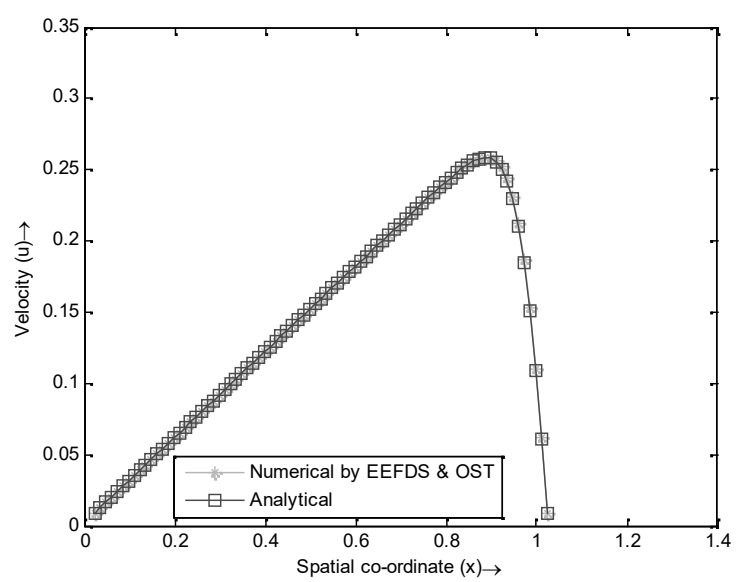

Figure 6: Solutions at $\tau=3.0$ for $\operatorname{Re}=100$ taking $h=0.0125, k=10^{-4}, a=2, b=4$.

\section{Error Estimation for Numerical Technique}

The accuracy of the scheme is measured in terms of the error norm defined by

$E=\left(\frac{\sum_{i=1}^{m}\left(\left|u_{i}^{\text {exact }}-u_{i}^{\text {computed }}\right|\right)^{2}}{\left.\left.\sum_{i=1}^{n}\right|_{i} ^{\text {exact }}\right|^{2}}\right)^{\frac{1}{2}}$

Where $u^{\text {exact }}$ and $u^{\text {computed }}$ represent exact and computed solutions respectively. 


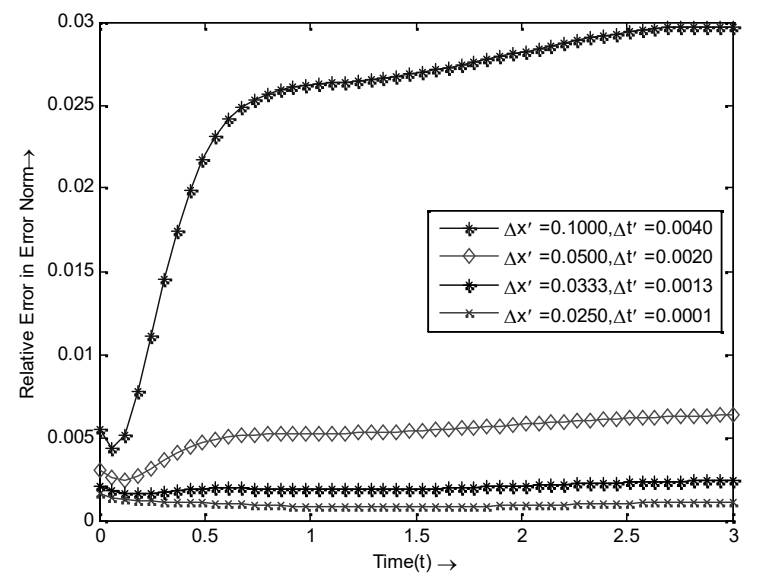

Figure 7: Error estimation for Expo FDS taking $L=1, t=3, R e=10$.

From figure 7 it is observed that at $t=0.5$ relative errors lie between $1.07 \times 10^{-3}$ and $2.17 \times 10^{-2}$ which show a $95.09 \%$ decrease. At $t=1$ relative error are $96.80 \%$ less which can be investigated since at this stage relative errors fall from $2.62 \times 10^{-2}$ to $8.36 \times 10^{-4}$. At $t=1.5$ relative errors lie between $8.00 \times 10^{-4}$ and $2.68 \times 10^{-2}$ and at this stage we investigate a $97.02 \%$ decrease in relative errors. At $t=2$ relative errors fall down from $2.81 \times 10^{-2}$ to $9.00 \times 10^{-4}$ which demonstrate a $96.79 \%$ go down. At $t=2.5$ relative errors lie between $1.03 \times 10^{-3}$ and $2.92 \times 10^{-2}$ which show a $96.50 \%$ decrease. Finally, at $t=3.0$ relative errors go down by $96.20 \%$ from $2.97 \times 10^{-2}$ to $1.13 \times 10^{-3}$ which are quite acceptable.

After computing relative errors we observe from figure 7 that our relative errors are quite acceptable and decreasing with respect to the smaller discretization parameters which show the convergence of the exponential explicit finite difference scheme. So, our explicit exponential finite difference scheme is consistent as well as stable.

Srivastava et al. [14] defined the rate of convergence of the scheme, computed using

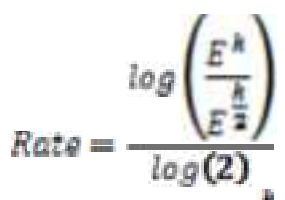

Where $E^{-h}$ and $E^{\frac{\pi}{2}}$ are the errors with the grid size $\mathrm{h}$ and $\frac{\boldsymbol{n}}{\mathbf{2}}$ respectively, is also shown in table 6 for Expo FDS.

Table 6 : Errors and rate of convergence at $R e=10, n=30000, t=3,0, a=2, b=4$.

\begin{tabular}{|l|c|c|}
\hline & \multicolumn{2}{|c|}{ Expo FDS } \\
\hline No. of Spatial steps $(\mathrm{m})$ & u-component error & Rate \\
\hline 5 & 0.149638346899101 & -
\end{tabular}




\begin{tabular}{|lll|}
\hline 10 & 0.033172483790659 & 2.173420990078319 \\
20 & 0.008016092653041 & 2.049015949043148 \\
40 & 0.001925644117421 & 2.057558083714238 \\
80 & $4.141848130038464 \mathrm{e}-04$ & 2.216994540074510 \\
\hline
\end{tabular}

From table 6, we observe that the Expo FDS used to evaluate numerical solutions for 1D NSE is second order accurate in space. From this table it can be seen that errors approach to zero as the mesh refines, which shows that the scheme is consistent.

\section{Conclusions}

In this paper, we have presented numerical solutions of 1D NSE with pressure gradient by using explicit exponential finite difference scheme for the reduced 1D NSE and Inverse OST. All the numerical results obtained by our method show reasonably good agreement with our analytical solutions and numerical solutions obtained based on CNS,E-EFDM, I-EFDM, FI-EFDM, RHC, and RPA. The numerical technique exhibits higher accuracy than RHC, RPA with which those are compared with the analytical solutions. Expo FDS is convergent and second order accurate in space. Therefore, it is concluded that the Expo FDS can be used to produce reasonably accurate numerical solutions of the governing equation at small times. The method is presented as an alternative method for solving a wide range of engineering problem.

\section{REFERENCES}

[1] Rusli, N., Kasiman, E.H., Hong, A.K., Yassin, Numerical computation of a two-dimensional NavierStokes equations using an improved finite difference method", Mathematika, 27(1), (2011), pp. 1-9.

[2] Azad M.A.K., Andallah L.S., An analytical solution of 1D Navier-Stokes equation, Int. J. Sc. Eng. Res., 5(2), (2014),pp. 1342-1348.

[3] Azad M.A.K., Andallah L.S., An explicit finite difference scheme for 1D Navier-Stokes equation, Int. J. Sc. Eng. Res., 5(3), (2014),pp. 873-881.

[4] Yang, T., McDonough J.M. Exact solution to Burgers' equation exhibiting erratic turbulent-like behavior, aiaa04.pdf.

[5] Orlowski A. and Sobczyk K., Rep Math. Phys., 7, (1989), pp. 59.

[6] Bhattacharya, M. C. An explicit conditionally stable finite difference equation for heat conduction problems. Int. J. Numer. Meth. Eng., 21, (1985), pp. 239-265.

[7] Bhattacharya, M. C., Finite difference solutions of partial differential Equations, Comm. Appl. Num. Meth., 6, (1990),pp. 173-184.

[8] Bahadır A. R., Exponential finite-difference method applied to Korteweg-de Vries equation for small times, Appl. Math. Comput., 160, (2005), pp. 675-682.

[9] Inan Bilge, Bahadir A.R. An explicit exponential finite difference method for the Burgers' equation, Euro. Int. J. Sc. Tech., 2(10), (2013), pp. 61-72.

[10] Kutluay, S., Bahadir, A. R., Özdeş, A. Numerical solution of one-dimensional Burgers' equation: explicit and Exact-explicit finite difference methods, J. Comput. Appl. Math., 103, (1999), pp. 251-261.

[11] Gülsu, Mustafa, ÖzişTurgut Numerical solution of Burgers' equation with restrictive Taylor approximation, Appl. Math. Comput., 171, (2005), pp. 1192-1200. 
[12] Gülsu, Mustafa, A finite difference approach for solution of Burgers' equation, Appl. Math. Comput., 175, (2006),pp. 1245-1255.

[13] Inan Bilge, Bahadir A.R., Numerical solution of the one-dimensional Burgers' equation: Implicit and fully implicit exponential finite difference methods, Pramana J. Phys., 81(4), (2013), pp. 547-556.

[14] Srivastava, V.K., Awasthi, M.K., Sing, S., An Implicit logarithmic finite- difference technique for twodimensional coupled viscous Burgers' equation, AIP Advances, 3, (2013), 122105 , pp.1-9. 


\section{Nomenclature}

\begin{tabular}{|c|c|c|c|}
\hline Symbols & Entities & Symbols & Entities \\
\hline$x, x^{\prime}$ & Cartesian co-ordinate & $R e$ & Reynolds number \\
\hline$t, t^{\prime}$ & Time & $u_{t}$ & $1^{\text {st }}$ order time derivative (Unsteady term) \\
\hline$L$ & Characteristic length & $u_{x}$ & $1^{\text {st }}$ order spatial derivative \\
\hline$U$ & Dimensionless horizontal velocity & $w u_{x}$ & Nonlinear convection term \\
\hline$u, u^{\prime}$ & Velocity along horizontal direction & $\frac{1}{R \varepsilon} u_{x i}$ & Diffusion term \\
\hline$p$ & Pressure & $m$ & Number of spatial steps \\
\hline$p_{x}$ & Pressure gradient in $x$-direction & $n$ & Number of time steps \\
\hline$p$ & Constant density & $u_{i}^{\prime n}$ & $\begin{array}{l}\text { Discrete approximation of } u^{\prime}\left(x^{\prime}, t^{\prime}\right) \text { at } \\
\text { the grid point }(h, n k)\end{array}$ \\
\hline$\mu$ & Dynamic viscosity of the fluid & $a, b$ & Constants \\
\hline v & Kinematic viscosity of the fluid & $\boldsymbol{n}, k$ & Spatial and time step size \\
\hline
\end{tabular}

